\title{
Performance Evaluation of Decomposition Methods in Perturbed Walking
}

\author{
Nurul Retno Nurwulan \\ Department of Industrial Engineering, Sampoerna University, Jakarta 12780, Indonesia
}

Corresponding Author Email: nurul.nurwulan@sampoernauniversity.ac.id

https://doi.org/10.18280/ria.340106

Received: 3 November 2019

Accepted: 20 January 2020

\section{Keywords:}

mobile phone, perturbed walking, decomposition, EEMD, wavelet, DWT, WPD

\begin{abstract}
Mobile phones cannot be separated from our daily lives. Most of young adults use their mobile phones while walking and this potentially cause accidents. The current study aimed to evaluate the performance of decomposition methods to detect perturbed walking. Ten young adults were asked to perform normal walking and walking while playing game on their phones. The vertical acceleration data were decomposed using 4, 6, and 8 levels of ensemble empirical mode decomposition (EEMD), discrete wavelet transform (DWT), and wavelet packet decomposition (WPD) before the calculation of the step stability index (SSI) to detect the perturbation during walking. Machine learning techniques were used to evaluate the performance of the decomposition methods. The evaluation results showed that DWT with 6 levels of decomposition outperformed other decomposition methods. In conclusion, the DWT can be used to increase the sensitivity of the SSI method in detecting walking perturbation.
\end{abstract}

\section{INTRODUCTION}

Young adults often use their mobile phones to send text messages or play games anytime and anywhere. It has become a habit that is normally done every day. Especially, after a popular augmented reality mobile game called Pokemon Go was launched. This game requires players to search around the city for wild animals to be trained as pets. The wild animals could appear any time and the players need to catch them to level up. Although this game is beneficial to encourage people to exercise, it is rather dangerous when people do not really pay attention to the road [1]. Mobile phone use during walking has a negative impact on working memory that increases perturbation that may cause accidents [2-4].

Healthy young adults have high flexibility to maintain their balance. However, it is important to know their limit in order to prevent injuries and/or accidents. Postural stability determination can be done using subjective methods such as questionnaires and rating scales. The time-up-and-go test and the Berg balance scale are the common prediction tools to predict the risk of falling that require subjective observation $[5$, $6]$.

Accelerometers have been widely used as the objective method to distinguish postural transition in physical activity. The acceleration data can reflect the intensity and frequency of human movement by capturing the external force proportionally [7]. Accelerometers can also show the velocity and displacement information [8]. Thus, postural sway and perturbation can be evaluated objectively using acceleration data. The evaluation results from acceleration data can be used as an early warning to raise awareness of how using mobile phone while walking may cause accidents.

Acceleration data analysis using pattern recognition method can be used to classify human movement data. The step stability index (SSI) was introduced as a measure to classify human movement. This index could distinguish non-fallers (healthy subjects) from the fallers [9]. The SSI was able to distinguish the movement of healthy subjects from the patients with postural stability issues. However, this index might not be as sensitive in classifying the postural sway of young and healthy subjects.

The SSI was developed by utilizing the intrinsic mode functions (IMFs) of the ensemble empirical mode decomposition (EEMD). The EEMD was proposed to overcome the mode mixing issues of the EMD [10]. The EEMD has been successfully applied in many fields because of its adaptability in analyzing nonlinear and non-stationary signal data. However, there are other decomposition methods that that are often used in signal processing, such as decomposition using discrete and continuous wavelet transforms. Past study used discrete wavelet transform (DWT) to distinguish walking on level ground and on a stairway [11]. Wavelet transforms have been proven to be more powerful than the EMD [12]. A comparison study concluded that continuous wavelet transform (CWT) was better for pattern recognition than EEMD and DWT [12].

The current study aimed to evaluate the performance of EEMD compared to discrete and continuous wavelet transforms in decomposing walking signal data. The results of decompositions were then used to calculate the SSI of the walking data. The performance evaluation of the decomposition methods was done using machine learning techniques: Fisher's linear discriminant analysis (LDA), artificial neural network (ANN), k-Nearest neighbors (KNN), support vector machines (SVM), and random forest (RF). The purpose of this performance evaluation was to get a better understanding of which decomposition method is more sensitive in recognizing human movement transition.

This paper is organized as follows. Section 2 describes the methods in conducting the experiment protocol and a brief description of the theoretical background behind SSI, EEMD, wavelet transform methods, and machine learning techniques. 
Section 3 presents results and discussion. Finally, the conclusion is presented in section 4 .

\section{METHODS}

Ten young adults $(24 \pm 0.94$ years $)$ were recruited. All subjects were free of postural stability-related disorder based on self-reports. The current study is approved by the IRB and informed consent forms were obtained from all subjects before their participation. The subjects were asked to perform walking and walking while playing games on their phones for 60 s each. Wearable accelerometers with sampling frequency of $30 \mathrm{~Hz}$ were attached on the subjects' ankle and knee (Figure 1). The accelerometers with sampling frequency of $30 \mathrm{~Hz}$ were chosen based on the results of preliminary study. In the preliminary study, wearable accelerometers of 20,30, and 100 $\mathrm{Hz}$ were evaluated by comparing their step count deviations from the real step counts. Although frequency of $30 \mathrm{~Hz}$ is considered as low, the preliminary results showed this frequency yielded the least deviation [13]. Further, a frequency rate of $30 \mathrm{~Hz}$ is enough to capture the gait signal data, as previous study used frequency rate of $20 \mathrm{~Hz}$ [14].

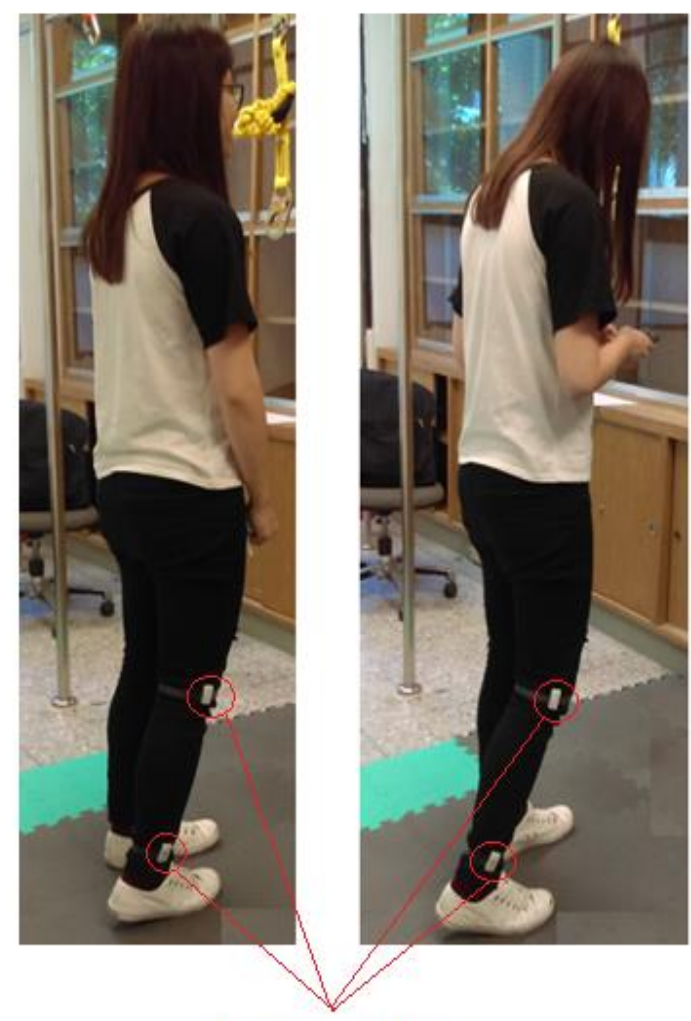

Wearable accelerometer

Figure 1. Normal walking (left) and walking while playing game on phone (right)

\subsection{Ensemble empirical mode decomposition}

Although the empirical mode decomposition (EMD) has been widely used due to its adaptability and efficiency in decomposing nonlinear and non-stationary signals, it has its drawback regarding the mode mixing issues. The EMD requires sufficient amplitude and frequency for decomposition analysis. Otherwise, the sifting process will derive an intrinsic mode function (IMF) with single tone modulated in amplitude instead of a superposition of two unimodular tones. The mode mixing phenomenon occurs when a single IMF either consists of oscillations with different frequencies or a signal with similar frequency is in different IMF components. It could imply either a single IMF consisting of signals of dramatically disparate scales or a signal of the same scale appearing in different IMF components. The mode mixing can change the physical meaning of each IMF component by replacing the part of IMF and driving it to the next IMF. Thus, it can falsely suggest that different physical processes may exist in an IMF. The ensemble empirical mode decomposition (EEMD) was proposed to overcome the mode mixing issues in the EMD [10].

Sifting process in the EMD method is done to extract the IMFs. It starts from identifying the extrema of the signal $\mathrm{X}(\mathrm{t})$ and then interpolate the local extrema using cubic spline to obtain the $\mathrm{U}(\mathrm{t})$ known as upper envelope and the $\mathrm{L}(\mathrm{t})$ known as lower envelope. After that, the local mean value of the upper and lower envelope can be calculated. Then the original signal is subtracted by the local mean value. Ultimately, the signal is replaced and this iteration is repeated until the obtained signal satisfies the condition that the number of extrema and the number of zero crossings must be either equal or differ at most by one, and the mean value of the envelopes is equal to zero in any point.

The main difference between EMD and EEMD is the addition of white noises in EEMD. A new time series data $\mathrm{Y}_{\mathrm{n}}(\mathrm{t})$ are generated by adding a white noise time series $\mathrm{u}_{\mathrm{n}}(\mathrm{t})$ to the original signal $\mathrm{X}(\mathrm{t})$

$$
Y_{n}(t)=X(t)+u_{n}(t)
$$

with $\mathrm{n}=1,2, \ldots, \mathrm{N} . \mathrm{N}$ is the ensemble number.

Using the same method as EMD, the new time series data are decomposed into a set of IMFs with a residual.

$$
Y_{n}(t)=\sum_{m=1}^{M-1} I M F_{m}^{(n)}(t)+r_{M}^{(n)}(t)
$$

where, M-1 is the total number of the IMFs and $\mathrm{r}$ is the residual

Evaluating the standard error of the deviation between original signal and the sum of the IMFs, could be done by dividing the amplitude by the square root of the ensemble number. In this study, the ensemble number of 100 and white noises of an amplitude that is about 0.2 standard deviation of the original signals, were added as suggested in the previous study [10].

\subsection{Wavelet transform}

The wavelet transform has become an important tool for many biomedical image analysis applications due to its ability in localizing frequency analysis [15]. Wavelet transforms can perform variable time-frequency localization. The orthonormal wavelets with a finite support provide a powerful method to decompose time series data into a multiresolution hierarchy of different localized frequency channels. Thus, it could analyze time series data at several levels of resolution simultaneously.

In wavelet transform, the signals are decomposed into a set of basis functions by translating and dilating the mother wavelet function. These basis functions wave up and down across the axis, integrating to zero. Hence, they are called wavelets. Wavelets are highly effective in analyzing non- 
stationary signals for noise reduction [12]. The discrete wavelet transform (DWT) converts a discrete time signal to a discrete wavelet representation. It converts signal data into high-pass and low-pass wavelet coefficient series with different cut-off frequencies. The DWT analyzes time-series data as a linear combination of the sum of the products of the wavelet coefficients and mother wavelet.

$$
\operatorname{DWT}(\mathrm{j}, \mathrm{k})=\int_{-\infty}^{\infty} \mathrm{X}(\mathrm{t}) \frac{1}{\sqrt{\mid 2^{\mathrm{j} \mid}}} \psi\left(\frac{\mathrm{t}-2^{\mathrm{j}} \mathrm{k}}{2^{\mathrm{j}}}\right) \mathrm{dt}
$$

where, $\psi, 2^{\mathrm{j}}, 2^{\mathrm{j}} \mathrm{k}$, and $\mathrm{X}(\mathrm{t})$ are wavelet function, scaling, shifting parameters, and the signal data, respectively. In the continuous wavelet transform (CWT), the $2^{\mathrm{j}}$ and $2^{\mathrm{j}} \mathrm{k}$ can be replaced by $\mathrm{a}$ and $\mathrm{b}$ and the formula for the CWT is as the following.

$$
\operatorname{CWT}(\mathrm{a}, \mathrm{b})=\int_{-\infty}^{\infty} \mathrm{x}(\mathrm{t}) \frac{1}{\sqrt{|\mathrm{a}|}} \psi\left(\frac{\mathrm{t}-\mathrm{b}}{\mathrm{a}}\right) \mathrm{dt}
$$

The continuous wavelet transform, such as wavelet packet decomposition (WPD) discretizes scale finer than DWT. Hence, it gives better frequency resolution for the decomposed signal [6]. However, the drawback of WPD is that it is less stable for signal reconstruction. Conversely, the DWT is more efficient due to its ability to provide perfect reconstruction of the signal upon inversion. This is because its coefficients can be used to reproduce an exact signal within numerical precision. Past study found that the optimum number of decomposition levels in DWT is 6 , whereas the optimum decomposition levels for WPD is 4 [12].

\subsection{Step stability index}

The walking patterns of fallers from non-fallers could be discriminated using the step stability index (SSI) [9]. The EEMD with 8-modes of IMFs is used to decompose the vertical acceleration data in the SSI method. Subsequently, the standard deviations (SD) of the IMF1 to IMF4 are used to develop the index as follows:

$$
\mathrm{SSI}=\frac{\text { SDIMF4 }}{(\text { SDIMF1 }+ \text { SDIMF2 }+ \text { SDIMF3 })}
$$

Using the SSI method, the fallers could be distinguished from the non-fallers because they exhibited lower SSI values that indicated less stable walking pattern. This happened because of the energy, as measured by standard deviation, of the IMF components at the step frequency lower than the energy of higher frequency components that related to subtle perturbed walking. In the current study, all subjects were healthy young adults. However, it was assumed that the distraction from the games on mobile phone would cause perturbation that is normally faced by the fallers. The original SSI used IMFs from the EEMD method. For the wavelet methods, the wavelet coefficients were used as the substitute of the IMFs [12].

\subsection{Machine learning techniques}

Performance evaluation using machine learning techniques has been a current trend, due to its robust and comprehensive method to evaluate the accuracy of measurement methods. The current study used Fisher's linear discriminant analysis (LDA), artificial neural network (ANN), k-Nearest neighbors (KNN), support vector machines (SVM), and random forest (RF).

The LDA is used to find a linear combination which characterizes two or more classes of events. It determines the discriminant dimension in response-pattern space with the ration of between-class over within-class variance of the data which is maximized [16]. The LDA is closely related to naïve Bayes since both classifiers assume Gaussian within-class distributions.

The ANN can be understood as parallel-distributed processing system. It has been widely used for pattern recognition due to its self-learning, adaptability, and robustness. The current study used multilayer perception (MLP) as it is a powerful neural network [17]. The MLP is a supervised learning technique that consists of at least an input layer, a hidden layer, and an output layer. It can distinguish data that are not linearly separable.

The KNN is an instance-based supervised classifier. All raw data (all instances) are put in a database and the corresponding output is determined based on the distance (similarity) calculation between instances. It is fast and simple because it does not require learning process [18]. The distance between training set and new observation is used to classify the data, whereas the new observation is assigned to the most similar class through the most votes of its k-nearest neighbors [16]. Each training pattern serves as a separate reference pattern with the nearest patterns determined by the Euclidean distance. The KNN selects the class of maximum posterior probability based on a nonparametric local probability. To the contrary of the LDA, KNN does not assume a particular shape of the distribution. Past studies using KNN as the classifier in human activity recognition have shown a satisfactory classification result [19].

The SVM can efficiently perform both linear and non-linear classifications. The linear SVM places a decision hyperplane in pattern space to classify test patterns into 2 classes. The difference between LDA and linear SVM is in its criterion, the linear SVM chooses the hyperplane with maximum margin [16]. Misclassifications are expected to happen in a few training data points when they cannot be separated perfectly. The nonlinear SVM can be created by restructuring the data using a kernel function to define a higher-dimensional alternative space. This kernel function maps the original data space to the higher-dimensional space.

The RF is one of the decision-tree's classifiers that improves the classification performance of a single-tree classifier by combining the bootstrap aggregating method and randomization in the selection of segmenting data nodes in the construction of decision tree $[19,20]$. In a comparative study, RF outperformed the other classifiers [20].

\section{RESULTS AND DISCUSSION}

The aim of the current study was to evaluate the performance of the decomposition methods to detect perturbation during walking. The SSI method was proposed to detect the postural sway of fallers while walking [9]. However, the comparison study by Alikovic et al. [12] showed that wavelets performed better than the EMD. Although SSI used EEMD, the EMD without mode mixing issues, it may not be 
better than wavelets in decomposing the signal data. The current study compared the performance of EEMD, DWT, and WPD using machine learning techniques.

The vertical acceleration data from the accelerometer were decomposed using EEMD, DWT, and WPD. The standard deviations were computed to get the values of the SSI. The original SSI uses IMF1 to IMF4 to calculate the SSI. As for the decomposition using wavelets, the coefficients were treated as the IMFs in EEMD [12]. In the current study, decomposition levels of 4,6 , and 8 were chosen to evaluate which decomposition level yields the highest accuracy.

Table 1. Performance accuracy of decomposition methods

\begin{tabular}{cccccc}
\hline \multicolumn{7}{c}{ LDA } & ANN & KNN & SVM & RF \\
\hline 4 Level decomposition & & & \\
\hline EEMD & & & & & \\
Ankle & 51.11 & 53.33 & 44.44 & 42.22 & 55.56 \\
Knee & 60.00 & 51.11 & 51.11 & 48.89 & 48.89 \\
DWT & & & & & \\
Ankle & 64.44 & 71.11 & 62.22 & 66.67 & 60.00 \\
Knee & 68.89 & 60.00 & 60.00 & 64.44 & 64.44 \\
WPD & & & & & \\
Ankle & 68.89 & 71.11 & 56.67 & 66.67 & 71.11 \\
Knee & 46.67 & 54.44 & 53.33 & 53.33 & 53.33 \\
\hline 6 Level decomposition & & & \\
\hline EEMD & & & & & \\
Ankle & 66.67 & 60 & 40 & 57.78 & 53.33 \\
Knee & 64.44 & 73.33 & 64.44 & 64.44 & 71.11 \\
DWT & & & & & \\
Ankle & 80 & 80 & 66.67 & 71.11 & 75.56 \\
Knee & 55.56 & 60 & 48.89 & 55.56 & 55.56 \\
WPD & & & & & \\
Ankle & 70 & 73.33 & 68.89 & 71.11 & 64.44 \\
Knee & 46.67 & 48.89 & 46.67 & 60 & 51.11 \\
\hline 8 Level decomposition & & & \\
\hline EEMD & & & & & \\
Ankle & 68.89 & 64.44 & 62.22 & 73.33 & 60.00 \\
Knee & 68.89 & 68.89 & 44.44 & 53.33 & 42.22 \\
DWT & & & & & \\
Ankle & 66.67 & 73.33 & 71.11 & 75.56 & 73.33 \\
Knee & 62.22 & 60.00 & 51.11 & 64.44 & 60.00 \\
WPD & & & & & \\
Ankle & 64.44 & 64.44 & 71.11 & 68.89 & 71.11 \\
Knee & 44.44 & 60.00 & 53.33 & 53.33 & 53.33 \\
\hline
\end{tabular}

The LDA, ANN, KNN, SVM, and RF were chosen as classifiers for the performance evaluation of the decomposition methods. As shown in Table 1, the DWT with 6 level of decomposition outperformed the other level of decompositions. The LDA and ANN as classifiers yielded the highest accuracy compared to the other methods for ankle data $(80 \%)$. Whereas, the EEMD with 6 level of decomposition performed the best for knee data based on the ANN classifier (73.33\%). Overall, the ANN performed better than other classifiers in all decomposition methods in both ankle and knee. The MLP was used for the ANN measure in the current study. It is able to find relation between features and could distinguish data that are difficult to separate linearly. The signal data of walking while playing game might be difficult to be distinguished from normal walking data by the other classifiers because the postural sway in healthy subjects may not be as obvious as in fallers.

Generally, the DWT performed better than other decomposition methods. To the contrary of the study by Alikovic et al. [12], the current study found that the DWT was better than the WPD. This is potentially because the nature of brain and gait signals are different. Previous comparative study evaluated the performance of wavelets as decomposition methods in walking detection showed that discrete wavelet was more efficient than the continuous wavelet [14]. Further, the current study used sampling frequency of $30 \mathrm{~Hz}$, whereas the study by Alikovic et al. [12] used sampling frequency of $256 \mathrm{~Hz}$. The DWT performed better than the WPD potentially because the current study used low sampling rate [14]. The DWT is a perfect reconstruction filter bank and suitable for low frequency data. The results of the study might be different if the sampling frequency was higher. However, the wearable accelerometer in the current study is affordable and more reliable compared to other wearable accelerometer with sampling frequency of $100 \mathrm{~Hz}$ [14]. In the comparative study of wearable and MEMS accelerometers, the wearable accelerometer outperformed the higher frequency and the MEMS accelerometers in terms of step detection accuracy [15].

The performance of ankle data was better than the knee data since the SSI used the vertical acceleration data for the analyses. Intuitively, the vertical displacement is more obvious when observed from the ankle than the knee. In general, the ankle data resulted in higher performance accuracy than the knee data. However, the highest accuracy was only $80 \%$. This could happen because the SSI method only utilized the vertical acceleration data. Whereas, the perturbation could happen in any axis, not only the vertical one. Thus, the performance of the decomposition methods may increase if the SSI method utilized not only the vertical acceleration data. Further, the SSI was developed to differentiate fallers and non-fallers. Conversely, the subjects in the current study were all healthy young adults with high adaptability to changes in the environment [3]. The performance accuracy could be higher if the acceleration data in all axes were considered and the subjects were not only healthy young adults [21].

\section{CONCLUSION}

While walking, young adults often play with their mobile phones to text messages, watch videos, read news, and/or play games. Doing another activity while walking has a negative impact on working memory and potentially increase the chance of injuries or even accidents. Despite the ability of young adults to quickly adjust themselves to the environment, it is important to know the limit of their balance ability. The current study used the SSI to calculate the perturbation during walking. The SSI has been used to distinguish non-fallers (healthy subjects) from the fallers. However, the postural sway during perturbed walking in healthy subjects might not be as easy to recognize.

The aim of this study was to evaluate the performance of decomposition methods. The current study used EEMD, DWT, and WPD for the signal decompositions. Machine learning techniques with LDA, ANN, KNN, SVM, and RF as classifiers were used to evaluate the performance of the decomposition methods. The evaluation results showed that the accuracy of ankle data was better than the knee data. The results suggest that ankle was a better location to place the wearable sensor since the SSI used the vertical acceleration data. Intuitively, it is easier to observe the vertical acceleration on ankle than on knee while walking. As for the decomposition methods, DWT outperformed EEMD and WPD with LDA and 
ANN as classifiers which implies using DWT as decomposition method potentially will increase the performance of the SSI in detecting walking perturbation in healthy subjects.

The limitation of this study was using accelerometers with low sampling rate that might affect the accuracy of the decomposition methods because DWT is more suitable for low sampling rate. Future studies utilizing higher sampling rate and higher decomposition level might confirm whether the DWT is still the best decomposition method.

In conclusion, the results of the current study suggest that decompositions using wavelet performed better than the EEMD in walking detection, with the DWT outperformed the WPD. The finding of the current study is important for the future studies in relation to determining postural sway caused by walking perturbation in healthy subjects.

\section{REFERENCES}

[1] Barbieri, S., Vettore, G., Pietrantonio, V., Snenghi, R., Tredese, A., Bergamini, M., Feltracco, P. (2017). Pedestrian inattention blindness while playing Pokémon Go as an emerging health-risk behavior: A case report. Journal of Medical Internet Research, 19(4): e86. https://doi.org/10.2196/jmir.6596

[2] Lamberg, E.M., Muratori, L.M. (2011). Cell phones change the way we walk. Gait and Posture, 36(3): 688690. https://doi.org/10.1016/j.gaitpost.2011.12.005

[3] Nurwulan, N.R., Jiang, B.C., Iridiastadi, H. (2015). Posture and texting: Effect on balance in young adults. PLOS ONE, 10: e0134230. https://doi.org/10.1371/journal.pone.0134230

[4] Nurwulan, N.R., Jiang, B.C. (2016). Possibility of using entropy method to evaluate the distracting effect of mobile phones on pedestrians. Entropy, 18: 390. https://doi.org/10.3390/e18110390

[5] Berg, K., Wood-Dauphinée, S., Williams, J.I., Gayton, D. (1989). Measuring balance in the elderly: Preliminary development of an instrument. Physiotherapy Canada, 41: 304-311. http://dx.doi.org/10. 3138/ptc.41.6.304

[6] Podsiadlo, D., Richardson, S. (1991). The timed "up \& go": A test of basic functional mobility for frail elderly persons. Journal of the American Geriatrics Society, 39: $142-148$

http://dx.doi.org/10.1111/j.15325415.1991.tb01616.x

[7] Yang, C.C., Hsu, Y.L. (2010). A review of accelerometry-based wearable motion detectors for physical activity monitoring. Sensors, 10: 7772-7788. https://doi.org/10.3390/s100807772

[8] Chen, K.Y., David R Bassett, J.R. (2005). The technology of accelerometry-based activity monitors: Current and future. Medicine and Science in Sports and Exercise, 37: 490-500.

[9] Cui, X., Peng, C.K., Costa, M.D., Weiss, A., Goldberger, A.L., Hausdorff, J.M. (2014). Development of a new approach to quantifying stepping stability using ensemble empirical mode decomposition. Gait and Posture, 39: 495-500 https://doi.org/10.1016/j.gaitpost.2013.08.036

[10] Wu, Z., Huang, N.E. (2009). Ensemble empirical mode decomposition: A noise-assisted data analysis method. Advances in Adaptive Data Analysis, 1(1): 1-41. https://doi.org/10.1142/S1793536909000047

[11] Sekine, M., Tamura, T., Togawa, T., Fukui, Y. (2000). Classification of waist-acceleration signals in a continuous walking record. Medical Engineering and Physics, 22(4): 285-291. https://doi.org/10.1016/s13504533(00)00041-2

[12] Alikovic, E., Kevric, J., Subasi, A. (2018). Performance evaluation of empirical mode decomposition, discrete wavelet transform, and wavelet packed decomposition for automated epileptic seizure detection and prediction. Biomedical Signal Processing and Control, 39: 94-102. https://doi.org/10.1016/j.bspc.2017.07.022

[13] Nurwulan, N.R., Chen, Y., Jiang, B.C. (2017). Activity recognition using wearable accelerometers. Proceedings of the 17th Asia Pacific Industrial Engineering \& Management Systems Conference Yogyakarta, Indonesia.

[14] Barralon, P., Vuillerme, N., Noury, N. (2006). Walk detection with a kinematic sensor: Frequency and wavelet comparison. Proceedings of the 28th IEEE EMBS Annual International Conference New York City, USA, pp. 1711-1714. https://doi.org/10.1.1.688.1812

[15] Laine, A.F. (2000). Wavelets in temporal and spatial processing of biomedical images. Annual Review of Biomedical Engineering, 2(1): 511-550. https://doi.org/10.1146/annurev.bioeng.2.1.511

[16] Duda, R.O., Hart, P.E., Stork, D.G. (1999). Pattern Classification. John Wiley \& Sons: Malden, MA, USA.

[17] Breiman, L., Friedman, J., Stone, C.J., Olshen, R.A. (1984). Classification and Regression Trees. CRC Press: Boca Raton, FL, USA.

[18] Zhang, T., Wang, J., Xu, L., Liu, P. (2006). Using wearable sensor and NMF algorithm to realize ambulatory fall detection. In Advances in Natural Computation, Springer: Berlin, Germany, 488-491.

[19] Bedogni, L., di Felice, M., Bononi, L. (2012). By train or by car? Detecting the user's motion type through smartphone sensors data. Proceedings of the 2012 IFIP Wireless Day (WD), Dublin, Ireland, pp. 1-6.

[20] Maroco, J., Silva, D., Rodrigues, A., Guerreiro, M., Santana, I., de Mendonça, A. (2011). Data mining methods in the prediction of Dementia: A real-data comparison of the accuracy, sensitivity and specificity of linear discriminant analysis, logistic regression, neural networks, support vector machines, classification trees and random forests. BMC Research Notes, 4(1): 299. https://doi.org/10.1186\%2F1756-0500-4-299

[21] Nurwulan, N.R., Jiang, B.C., Novak, V. (2019). Development of postural stability index to distinguish different stability states. Entropy, 21(3): 314. https://doi.org/10.3390/e21030314

\section{NOMENCLATURE}

a

$\mathrm{b}$

ANN

CWT

DWT

EEMD

EMD

IMF

KNN

LDA scaling in continuous wavelet shifting parameter in continuous wavelet artificial neural network continuous wavelet transform discrete wavelet transform ensemble empirical mode decomposition empirical mode decomposition intrinsic mode function k-nearest neighbors linear discriminant analysis 


$\begin{array}{llll}\text { M } & \text { total number of IMFs } & \mathrm{Y}_{\mathrm{n}}(\mathrm{t}) & \text { time series data } \\ \mathrm{MLP} & \text { multi layer perception } & \text { Greek symbols } & \\ \mathrm{N} & \text { ensemble number } & & \\ \mathrm{r} & \text { residual } & \psi & \text { wavelet function } \\ \mathrm{RF} & \text { random forest } & \text { Subscripts } & \\ \mathrm{SD} & \text { standard deviation } & & \\ \mathrm{SSI} & \text { step stability index } & 2^{\mathrm{j}} & \text { scaling in discrete wavelet } \\ \mathrm{SVM} & \text { support vector machine } & 2^{\mathrm{j}} \mathrm{k} & \text { shifting parameter in discrete wavelet } \\ \mathrm{u}_{\mathrm{n}}(\mathrm{t}) & \text { white noise time series } & & \end{array}$

$\mathrm{X}(\mathrm{t})$

original signal 